\title{
Outcome of Supracondylar Fractures of the Humerus in Children Treated by Closed Reduction and Percutaneous Pinning
}

\author{
Devkota P, ${ }^{1}$ Khan JA, ${ }^{1}$ Acharya BM, ${ }^{1}$ Pradhan NMS, ${ }^{1}$ Mainali LP, ${ }^{1}$ Singh M, ${ }^{1}$ Shrestha SK, ' Rajbhandari AP' \\ 'Department of Orthopaedic and Trauma Surgery, Patan Hospital, Lalitpur, Nepal
}

\begin{abstract}
Supracondylar fractures of humerus in children are common injuries. Displaced fractures are inherently unstable. Conservative treatment results in malunion. Open reduction and internal fixation (ORIF) is more invasive and recovery is prolonged. From September 2004 to September 2005, 102 displaced supracondylar fractures of humerus, aged between one and half year to 13 years, were treated using close reduction and percutaneous Kirschner $(\mathrm{K})$ wire fixation under c-arm fluoroscopy. Seventy nine patients were treated by cross K-wires and in twenty three cases lateral two K-wires were put. Above elbow plaster of paris back slab was applied in all cases for at least four weeks. Back slab, K-wires were removed after four weeks and elbow range of motion exercise was started. Results were analyzed using Flynn's criteria. All patients were followed up to $14^{\text {th }}$ week postoperatively. In cross $\mathrm{K}$-wire group(N=79) $70.8 \%$ had excellent, $22.7 \%$ good, $3.8 \%$ fair and $2.5 \%$ had poor results at eight weeks follow up which was improved to $91.1 \%$ excellent, 6.3 good, $1.2 \%$ fair and $1.26 \%$ poor results at 14 weeks follow up. In lateral K-wire group ( $\mathrm{N}=23) 70 \%$ had excellent, $21.7 \%$ good, $4.3 \%$ fair and $4.3 \%$ had poor result at eighth week which was improved to $91.3 \%$ excellent, $4.3 \%$ good, $4.3 \%$ fair and no poor result at $14^{\text {th }}$ week follow up. Eight patients got superficial pin tract infection and seven patients sustained ulnar nerve injury post operatively. We recommend this procedure for displaced supracondylar fractures in children as it is safe and cost effective procedure with acceptable complication rates.
\end{abstract}

Key words: closed reduction, humerus, percutaneous pinning, supracondylar fracture

\section{INTRODUCTION}

Supracondylar fracture of the humerus is the second most common fracture in children and is usually accompanied with marked swelling which presents a formidable challenge for reduction and immobilisation. ${ }^{1-}$ ${ }^{3}$ Many of these fractures are unstable after reduction except in an acutely-flexed position. If considerable swelling is present, the position may compromise with vascularity and predispose to Volkmann's ischemic contracture. ${ }^{4,5}$ Immobilization in the safer right-angle position will frequently allow the fragments to slip, producing various deformities causing supracondylar dilemma. ${ }^{6}$

Closed reduction and fixation with percutaneous Kirschner (K) wire was first described by Swenson. ${ }^{7}$ He pointed out the advantage as 1) stable fixation of fracture fragment, 2) decreased risk of circulatory compromise in the form of restoration of radial pulse in nearly 90 percent of cases of brachial artery injury, and 3) a simple and cost-effective procedure. The purpose of this study was to assess the ability of closed reduction and percutaneous $\mathrm{K}$-wire fixation, to obtain 
Devkota et al. Outcome of Supracondylar Fractures of the Humerus in Children Treated by Closed Reduction and ...

and maintain an adequate fixation, and to evaluate the recovery of elbow range of motion (ROM) and carrying angle.

\section{MATERIAL AND METHODS}

This is a prospective, observational and clinical study done at the department of Orthopedic and Trauma Surgery, Patan Hospital, from September, 2004 to September, 2005. A total of 102 children with fractures supracondylar of humerus Grade III, (Figure 1) closed or Grade I open were included after taking informed consent. $^{2,8}$ The patients were aged between one-anda-half to 13 years with the mean age of 7.76 years. We excluded Grade I and Grade II fractures and open fractures of more than Grade I. bilateral fractures and those cases which had previous contralateral fractures around the elbow were also excluded. The time of operation ranges from the first day of injury to the eighth day of injury with the mean time of operation being 4.6 days. The patients were evaluated as described by Flynn and the results compared with the contralateral normal elbow. ${ }^{9}$

Under general anesthesia, using c-arm fluoroscopy closed reduction was done. The forearm was then pronated and the elbow acutely flexed and held temporarily by adhesive tape. Pronation de-rotates the distal fragment from its frequently medially rotated position and locks it in correct alignment. ${ }^{10}$ When satisfactory reduction had been achieved then fixation was done by two cross $\mathrm{K}$ wires of 1.5 or $2.0 \mathrm{~mm}$ size (Figure 2).

Sometimes the operating surgeons were not clear about the medial side landmark, and then two parallel lateral Kwires were put. The pins were bent and cut off outside the skin and a well-padded, above-elbow, posterior back-slab was applied. The elbow could be held in any position without losing the reduction, and the optimum position, usually 60 to 90 degrees of elbow flexion, allowed free blood flow. The patient was carefully observed for twelve to seventy-two hours (average 58 hours) and then discharged. The above-elbow plaster of paris (POP) back slab was kept for four weeks and the pins and slab were removed in the out-patient (OPD) clinic. Elbow ROM was started after removing the POP back slab.

The follow-up was arranged as follows: the first followup on the seventh day to inspect the wound; the second follow-up on the second week for wound inspection or suture removal and to see the pin configuration; the third follow-up on the fourth week for the removal of plaster slab and pins and to start physiotherapy; the fourth follow-up on the eighth week post-operatively to see the ROM and carrying angle of the elbow; and the final follow-up on the fourteenth week post-operatively to see the final result of the study (Figure 3,4). All the statistical data were analyzed with Microsoft excel.

\section{RESULTS}

Male children were affected more than female with the ratio being 58 to 44 . The left side was affected more than the right (ratio 54:48). The extension type was $95(93.13 \%)$ and flexion type $7(6.86 \%)$. Twenty three $(22.55 \%)$ cases were treated by two lateral parallel K-wires and seventy nine $(77.45 \%)$ by cross K-wires. Preoperatively, three cases were open fractures, six cases had nerve injuries (median nerve three, ulnar nerve two and radial nerve one) and there were no cases of vascular injuries (Figure 5).

Post-operatively, seven patients $(6.86 \%)$ got ulnar nerve injury and eight $(7.84 \%)$ patients got pin tract infection, which was superficial and healed after removing pins and oral antibiotic administration. All except one nerve injuries recovered within three-and-ahalf months post-operatively. There was no ulnar nerve injury in the patient treated by inserting only lateral two K-wires (Figure 6, 7).

Callus formation was seen in all patients at the fourth week post-operatively before removing the K-wires, (Figure 3). The fracture united in all cases at the fourth week post-operatively. Results were analyzed using Flynn's criteria. ${ }^{9}$ All patients were followed at eighth week and the $14^{\text {th }}$ week postoperatively. In the crossed K-wire group ( $\mathrm{N}=79$ ), $70.8 \%$ had excellent, $22.7 \%$ good, $3.8 \%$ fair and $2.5 \%$ poor results at the eight week follow-up which was improved to $91.1 \%$ excellent, 6.3 good, $1.2 \%$ fair and $1.26 \%$ poor results at the 14 week follow-up. In the lateral $\mathrm{K}$-wire group ( $\mathrm{N}=23), 70 \%$ had excellent, $21.7 \%$ good, $4.3 \%$ fair and $4.3 \%$ poor results at eight weeks which was improved to $91.3 \%$ excellent, $4.3 \%$ good, $4.3 \%$ fair and no poor result at the $14^{\text {th }}$ week follow-up (Table 2).

During this study, complications like vascular injury, compartment syndrome, myositis ossifications, significant mal-union and non-union were not encountered. Distal pin migration was seen in 4 $(3.92 \%)$ patients, loss of reduction was seen in 2 $(1.96 \%)$, which was not significant and did not require re-reduction and re-pinning.

\section{DISCUSSIONS}

A supracondylar fracture of the humerus is the most common fracture of the elbow in children. Unfortunately, it can also be one of the most difficult fractures to treat. While some authors have relied on a child's remodeling capability to compensate for inadequate reduction, most authors agree that accurate reduction with minimum joint and soft-tissue trauma is required to achieve the best possible functional result. ${ }^{11-13}$ 
Devkota et al. Outcome of Supracondylar Fractures of the Humerus in Children Treated by Closed Reduction and ...

In this study, the results of both lateral and cross pin insertion groups at eighth post-operative week showed excellent results in around seventy percent of patients. At the $14^{\text {th }}$ week post operatively these excellent results were seen in more than ninety percent of the cases. We believe that this increase of range of motion of the elbow was because of the physiotherapy. Those patients who had good or fair results were having severe soft tissue injuries or repeated closed reduction performed in another center. Khan obtained $88 \%$ excellent, four percent good and four percent poor results in his study. ${ }^{14}$ Tiwari observed 88 percent satisfactory results, among which $42 \%$ were excellent, in his series of late-presenting supracondylar fractures of humerus in children. ${ }^{15}$ These two studies are comparable to our study.

Cubitus varus deformity is the most common problem seen after the treatment of supracondylar fractures. The cause of the deformity is coronal rotation, or tilting of the distal fragment. ${ }^{16}$ Some investigators believed that varus deformity is due to epiphyseal growth disturbance or rotation of the distal fragment. ${ }^{17}$ Smith suggested that residual medial tilt after reduction is the most important factor in varus angulations, with isolated rotational deformities being corrected by compensatory rotation at the shoulder. ${ }^{18}$ This concept has become popular in understanding the sequel of alteration in carrying angle. ${ }^{19}$

In this series, six patients $(5.88 \%)$ had nerve injury preoperatively, out of which three had median, two ulnar and one radial. Seven patients got ulnar nerve injuries post-operatively, which is $6.86 \%$ of the total number. All the nerve injuries recovered within 14 weeks postoperatively except one case. The incidence of postoperative has been estimated to range from 5 to $19 \% .{ }^{20}$ Culp recommends that initial observation and supportive therapy for neural injury associated with a closed, displaced, supracondylar fracture of the humerus; and that if there is no clinical or electromyography evidence of return of neural function at five months after injury, exploration and neurolysis should be performed. If the nerve is in continuity, the prognosis after neurolysis is excellent. ${ }^{21}$

In the present study, there was no incidence of ulnar nerve injury where pinning was done from the lateral side; and we did not find any difference in bonehealing and stability between lateral-pin insertion and cross-pin insertion as we followed the same treatment protocol for both the groups. Skaggs found that the use of lateral-entry pins alone was effective for even the most unstable supracondylar humeral fractures and they saw no iatrogenic ulnar nerve injuries, and no reduction was lost. ${ }^{22-23}$ They suggest that the important technical points for fixation with lateral-entry pins are (i) maximizing separation of the pins at the fracture site, (ii) engaging the medial and lateral columns proximal to the fracture, (iii) engaging sufficient bone in both the proximal segment and the distal fragment, and (iv) maintaining a low threshold for use of a third lateral entry pin if there is concern about fracture stability or the location of the first two pins.

In the present series, eight $(7.84 \%)$ patients developed pin-tract infections, which were superficial and healed after removing pins and administration of oral antibiotics. No deep infection or septic arthritis was found. Pirone found superficial pin-tract infection in two percent of cases with no deep infection and septic arthritis. ${ }^{24}$ We had more pin tract infection which was probably because of poor hygienic conditions of the patient. In the present series, the distal pin migration was seen in four $(3.92 \%)$ patients and loss of reduction in two $(1.96 \%)$, which were not significant and so required no re-reduction and re-pinning. Gordon observed pin-tract migration in six percent of cases and Lee noticed the loss of reduction in seven percent of cases. ${ }^{25-26}$

\section{CONCLUSIONS}

Closed reduction and percutaneous $\mathrm{K}$-wire pinning in the management of supracondylar fractures of the humerus in children is safe as regards avoidance of vascular complications, effective in obtaining good results, and relatively economical regarding hospitalization. The disadvantage is the need for technical proficiency and the availability of c-arm fluoroscopy. There is a risk of injuring the ulnar nerve in cross pinning and this can be avoided by pinning only lateral two pins.

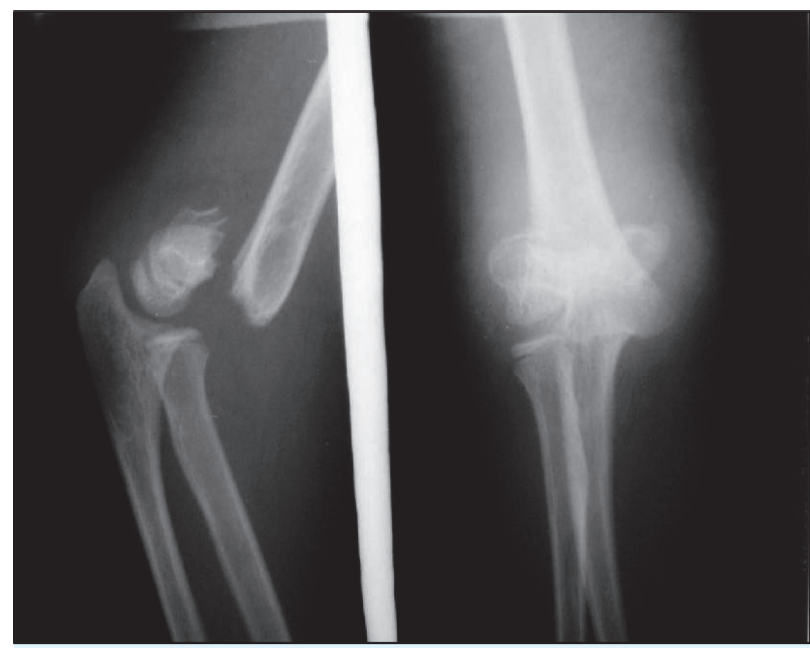

Figure 1. Anterio-posterior (AP) and lateral view of elbow showing Gartland Type III extension type of supracondylar fracture of humerus in an eight-year old child 
Devkota et al. Outcome of Supracondylar Fractures of the Humerus in Children Treated by Closed Reduction and ...

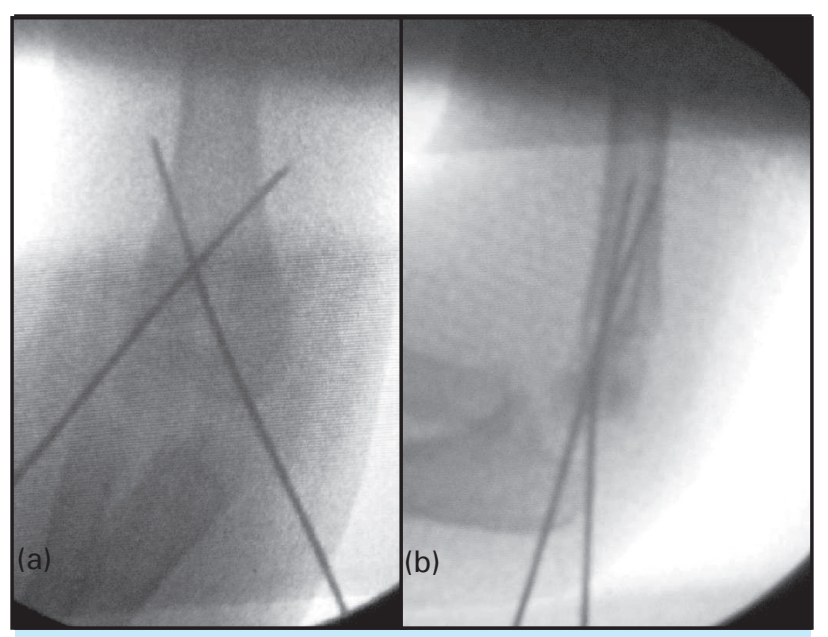

Figure 2. Intra-operative picture of c-arm after fixing with K-wire (a) anterior-posterior (AP) and (b) lateral view

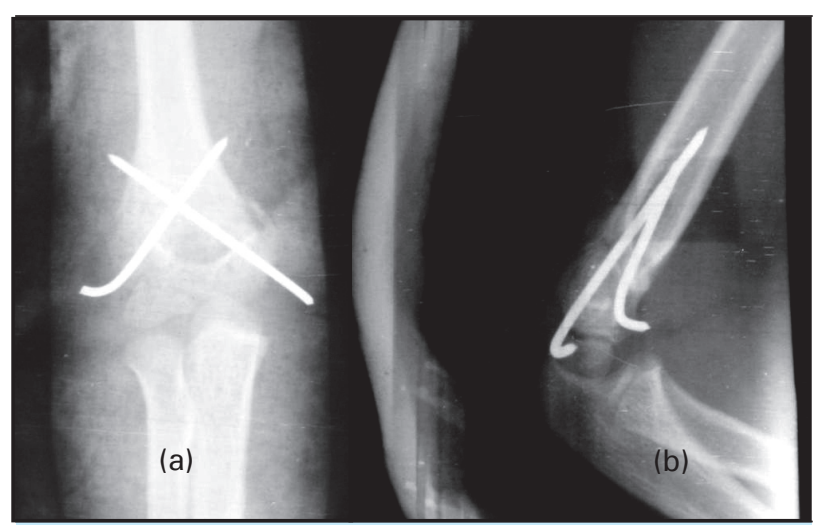

Figure 3. After K-wire fixation and POP back slab (a) anterior-posterior (AP) and (b) lateral view on fourth week post-operatively

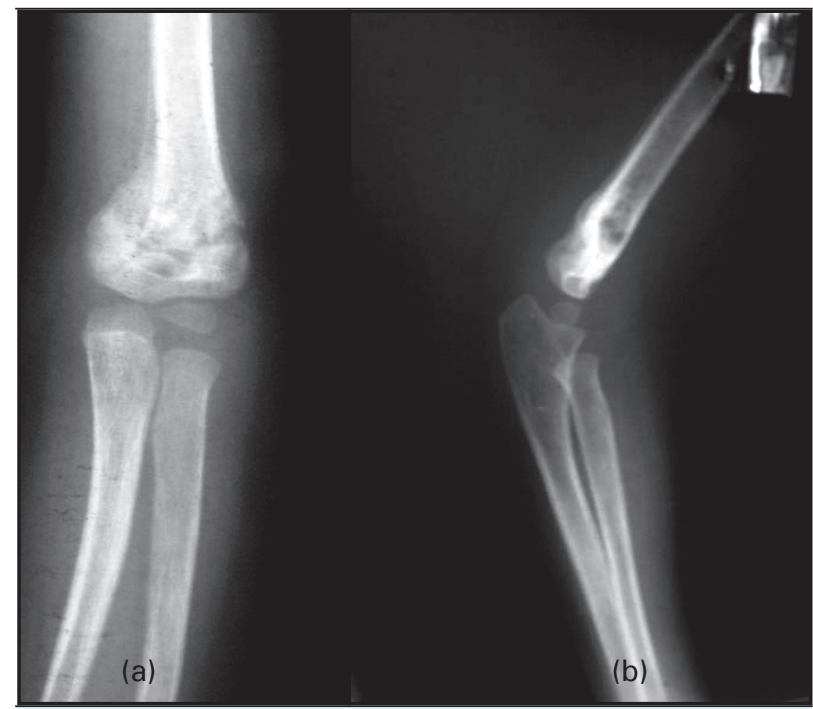

Figure 4. Good union seen after K-wire removal (a) anterior-posterior (AP) and (b) lateral view on the eighth week post-operatively
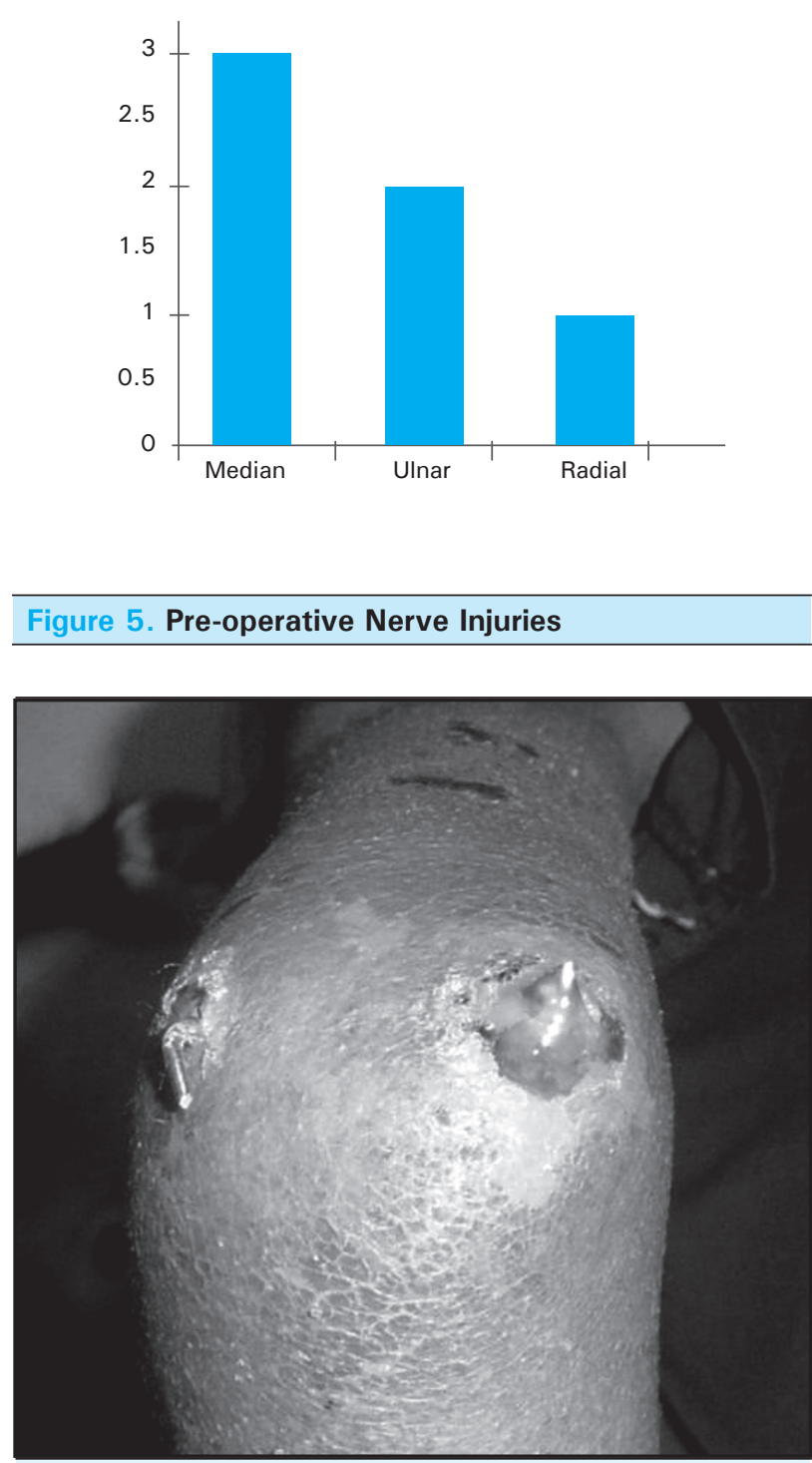

Figure 6. A typical pin tract infection

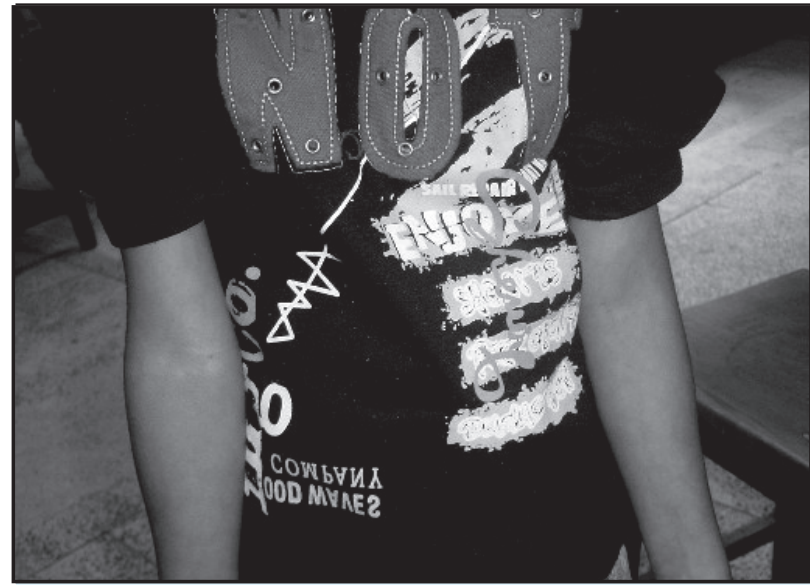

Figure 7. Comparison of carrying angle of both elbows shows almost similar results 
Devkota et al. Outcome of Supracondylar Fractures of the Humerus in Children Treated by Closed Reduction and ...

Table 1. Results of the Evaluation of the Hundred and Two Patients According to the Flynn Criteria

\begin{tabular}{|c|c|c|c|c|c|c|}
\hline \multirow{3}{*}{$\begin{array}{c}\text { Pin } \\
\text { Inserted }\end{array}$} & \multirow{3}{*}{$\begin{array}{l}\text { No. of } \\
\text { Cases }\end{array}$} & \multirow{3}{*}{$\begin{array}{l}\text { Follow up } \\
\text { duration* }\end{array}$} & \multirow{3}{*}{$\begin{array}{c}\text { Excellent } \\
\text { Number (\%) }\end{array}$} & \multicolumn{3}{|c|}{ Grading } \\
\hline & & & & Good & Fair & Poor \\
\hline & & & & Number (\%) & Number (\%) & Number (\%) \\
\hline \multirow[t]{2}{*}{ Cross } & 79 & 8 & $56(70.88)$ & $18(22.78)$ & $3(3.79)$ & $2(2.53)$ \\
\hline & & 14 & 72 (91.19) & $5(6.29)$ & $1(1.26)$ & $1(1.26)$ \\
\hline \multirow[t]{2}{*}{ Lateral } & 23 & 8 & $16(69.56)$ & $5(21.73)$ & $1(4.34)$ & $1(4.34)$ \\
\hline & & 14 & $21(91.3)$ & $1 \quad(4.34)$ & $1(4.34)$ & $0(0.0)$ \\
\hline
\end{tabular}

* week

\section{REFERENCES}

1. Takahara M, Sasaki I, Kimura T, Kato H, Minami A, Ogino T. Second fracture of distal humerus after cubital varus malunion of a supracondylar fracture in children. J Bone Joint Surg $\mathrm{Br}$ 1998;80(5):791-7.

2. Gartland JJ. Management of supracondylar fracture of humerus in children. Surg Gynecol Obstet 1959;109:145-54.

3. Mitchell WJ, Adams JP. Supracondylar fracture of humerus in Children; A Ten year review. J Am Med Assn 1961;175:573-7.

4. Herzenberg JE, Koreska J, Carroll NC, Rang M. Biomechanical testing of pin fixation techniques for pediatric supracondylar elbow fractures. Orthop Trans 1988;12:678-9.

5. Kallio PE, Foster BK, Paterson DC. Difficult supracondylar elbow fractures in children: analysis of percutaneous pinning technique. J Pediatr Orthop 1992;12:11-5.

6. Labelle H, Bunnell WP, Duhaime M. Cubitus Varus Deformity following supracondylar fracture of humerus in children. J Pediatr Orthop 1982;2:539-46.

7. Swenson AL. The treatment of supracondylar fracture of humerus by Kirschner Wire Transfixation. J Bone Joint Surg Am 1948;30:9937.

8. Gustilo RB, Anderson JT. Prevention of infection in the treatment of one thousand and twenty-five open fractures of long bones: retrospective and prospective analyses, J Bone Joint Surg Am 1976;58(4):453-8.

9. Flynn JC, Matthews JC, Benoit RJ. Blind pinning of displaced supracondylar fracture of humerus in children. Sixteens' years experience with long term follow up. J Bone Joint Surg Am 1974;56:263-72.

10. D'ambrosia RD. Supracondylar Fractures of the Humerus Prevention of Cubitus Varus. J Bone Joint Surg Am 1972;54:60-6.

11. Attenborough CG. Remodeling of the Humerus after Supracondylar Fractures in Childhood. J Bone Joint Surg Br 1953;35:386-95.

12. Boyd DW, Aronson DD. Supracondylar fractures of the humerus: a prospective study of percutaneous pinning. J Pediatr Orthop 1992;12:789-94.

13. Cheng JC, Lam TP, Shen WY. Closed reduction and percutaneous pinning for type III displaced supracondylar fractures of the humerus in children. J Orthop Trauma 1995;9:511-5.
14. Khan AQ, Goel S, Abbas M, Sherwani MK. Percutaneous Kwiring for Gartland type III supracondylar humerus fractures in children. Saudi Med J 2007; 28(4):603-6.

15. Tiwari A, Kanojia RK, Kapoor SK. Surgical management for late presentation of supracondylar humeral fracture in children. J Orthop Surg (Hong Kong) 2007;15(2):177-82.

16. Kallio PE, Foster BK, Paterson DC. Difficult supracondylar elbow fractures in children: analysis of percutaneous pinning technique. J Pediatr Orthop 1992;12:11-5.

17. Smith L. Deformity following Supracondylar Fractures of the Humerus. J Bone Joint Surg Am 1960;42:235-52.

18. Smith L. Supracondylar Fractures of the Humerus Treated by Direct Observation. Clin Orthop Relat Res 1967;50:37-42.

19. Mann TS. Prognosis in Supracondylar Fractures. J Bone Joint Surg Br 1963;45:516-22.

20. McGraw JJ, Akbarnia BA, Hanel DP. Neurological Complications Resulting from Supracondylar Fractures of the Humerus in Children. J Pediat Orthop 1986;6:647-50.

21. Culp RW, Osterman AL, Davidson RS, Skirven T. Neural injuries associated with supracondylar fractures of the humerus in children. J Bone Joint Surg Am 1990;72:1211-5.

22. Skaggs DL, Hale JM, Bassett J, Kaminsky C, Kay RM, Tolo VT. Operative treatment of supracondylar fractures of the humerus in children. The consequences of pin placement. J Bone Joint Surg Am 2001;83:735-40.

23. Skaggs DL, Cluck MW, Mostofi A, Flynn JM, Kay RM. LateralEntry Pin Fixation in the Management of Supracondylar Fractures in Children. J Bone Joint Surg Am 2004;86:702-7.

24. Pirone AM, Graham HK, Krajbich JI. Management of displaced extension-type supracondylar fractures of the humerus in children. J Bone Joint Surg Am 1988;70:641-50.

25. Gordon JE, Patton CM, Luhmann SJ, Bassett GS. Schoenecker PL. Fracture stability after pinning of displaced supracondylar distal humerus fractures in children. J Pediatr Orthop 2001;21:313 -8

26. Lee SS, Mahar AT, Miesen D, Newton PO. Displaced pediatric supracondylar humerus fractures: biomechanical analysis of percutaneous pinning techniques. J Pediatr Orthop 2002;22:440 -3. 